\title{
MONTANE MEADOW BUTTERFLY SPECIES DISTRIBUTIONS IN THE GREATER YELLOWSTONE ECOSYSTEM
}

\author{
JENNET C. CARUTHERS $\uparrow$ DIANE DEBINSKI \\ ECOLOGY, EVOLUTION \& ORGANISMAL BIOLOGY \IOWA STATE UNIVERSITY $\downarrow$ AMES
}

\begin{abstract}
$\downarrow \quad$ ABSTRACT
The composition of butterfly communities is a good indicator of changing environmental conditions. Butterflies have tight associations with the plant community due to their dependence on plants throughout their life history. These associations make butterfly distributions predictable based on the plant communities. Butterfly abundance data have been collected annually since 1997 within montane meadow sites characterized along a hydrologic gradient within the Greater Yellowstone Ecosystem. From this research, community composition may be predictable relative to future climatic changes and key habitat constraints. Identifying such variables is important for butterfly conservation.
\end{abstract}

\section{$\downarrow$ INTRODUCTION AND OBJECTIVES}

Butterfly communities have been established in the literature as well known indicators for environmental changes (Debinski and Brussard, 1994; Gutierrez and Menendez, 1998; Simonson et al., 2001; Dennis et al., 2006; Scalercio et al., 2006). Butterfly species richness can be predicted from models using landscape variables such as elevation and topographic heterogeneity (Nally et al., 2003) and plant richness (Field et al., 2006). Butterflies have been used to predict areas that represent hotspots of biological diversity (Simonson et al., 2001) and they may serve as good indicators of climate change (Debinski et al., 2000; Debinski et al.,
2006; Parmesan, 2006). They are thought to be good indicators because they play important roles within different functional groups, including herbivores, pollinators, and prey and their distribution patterns are correlated with habitat diversity (Scalercio et al., 2006). Conservation concerns have been heightened by long-term studies in Europe that have shown butterfly communities to be declining (Thomas and Albery, 1995; Grill and Cleary, 2003; and Binzenhofer et al., 2005). Understanding what is causing this decline could lead the way to uncoupling the loss of species diversity and protecting the diversity of other taxa that share the butterfly habitat.

There are many factors that have been identified to have a negative effect on butterfly communities. Loss of suitable habitat is one of the most threatening factors for butterfly species persistence (Grill and Cleary, 2003). Changes in habitat suitability due to climatic variations can also affect butterfly abundance (Ockinger et al., 2006). The pristine nature and minimal human impacts in the Greater Yellowstone Ecosystem make it an ideal location for studying the effects of climate driven variation on butterfly communities.

In this study we hope to determine whether the butterfly communities in the Greater Yellowstone Ecosystem fluctuate predictably relative to alterations of climate driven changes along a hydrologic gradient in the landscape. Climate driven variables include temperature and precipitation fluctuations, and the effects of such changes on species of forbs that serve as host 
plants and nectar resources for butterflies in this ecosystem. Shallow-rooted forbs are expected to be especially affected by an increase in temperature (Devalpine and Harte, 2001; and Saavedra et al., 2003; Cross and Harte, 2007) and a decrease in precipitation (Weaver, 1958) and we hypothesize that these changes will be reflected in butterfly abundance of the species that use these forbs for host plants or nectar. Stability in the butterfly community will be examined at different levels from a hierarchical perspective, including species presence and absences, abundance ranking, and absolute abundance (Pimm, 1984; Lawton and Gaston, 1989; Rahel, 1990). Groups of butterfly species using similar functional guilds of plants will be examined for correlated changes. Changes in floral resource cover and host plant percent cover will also be monitored to relate to changes in butterfly species abundance.

\section{$\downarrow$ METHODS}

\section{Study Area}

The Greater Yellowstone Ecosystem was divided into two study regions for our project, which will be referred to as the Gallatin and the Teton regions. The Gallatin region includes 30 sites within the Gallatin National Forest and the northwestern portion of Yellowstone National Park in Montana. The Teton region has 25 sites within the Grand Teton National Park and Bridger Teton National Forest in Wyoming. The Gallatin and Teton regions are separated by $192 \mathrm{~km}$, yet both have similar plant and butterfly communities (Su et al. 2004). The meadows selected for the surveys in both regions are approximately at the same elevation with homogenous topographic features. The average elevation for sites within the Gallatin region is $2098 \mathrm{~m}$, and $2120 \mathrm{~m}$ in the Teton region. The meadows range from $1-7861 \mathrm{ha}$, with an average meadow patch of 500 ha. Six meadow types with distinct plant species were characterized, M1-M6, along a hydrologic gradient (hydric to xeric respectively) using satellite imagery (Jakubauskas et al., 1996). The Gallatin region has five replicates of each meadow type from M1-M6, and the Teton region has five replicates of each meadow type except meadows characterized as M4, which are not represented in the Teton region. The meadows were characterized as suitable for survey sites if they were within $8 \mathrm{~km}$ from a road or trail, a minimum of $100 \mathrm{~m}$ by $100 \mathrm{~m}$ and no more than 2 $\mathrm{km}$ on a side, as well as at least $500 \mathrm{~m}$ from another meadow site (Debinski et al., 2001).

\section{Field Surveys}

Field surveys were conducted during June through August for two week periods at each region, alternating between the two regions. Two surveys for each region were completed annually by early August. These surveys are part of a long-term study that was initiated in 1997 and continued through 2007. Sites were located in the field using written directions, topographic maps, and GPS coordinates to find the site marker stake within the meadow. In 1997, two randomly selected cardinal directions $(\mathrm{NW}, \mathrm{NE}$, etc.) were used for the placement of a $50 \mathrm{~m}$ x 50 $\mathrm{m}$ plot. This plot was annually measured and flagged so that every year the same area was sampled. Surveys were conducted on sunny days when the temperature is above $70^{\circ} \mathrm{F}$ with low to moderate wind. The survey lasted for twenty minutes with two people surveying butterflies within the plot. Abundance data were collected by netting butterflies, collecting them in glassine envelopes, and finally releasing them at the end of the survey after individual butterflies were identified to the species level.

At each of the 55 study sites, vegetation was surveyed once per season in the middle of the growing season (July) in $20 \mathrm{~m} \times 20 \mathrm{~m}$ plots which also had one corner of two cardinal directions located at the site marker stake. Cover estimates to $1 \%$ resolution were made for ten most dominant forb species in each plot. Nectar resources were quantified by counting the number of racemes for all flowering plant species along a $1 \mathrm{~m}$ wide transect positioned diagonally across the $50 \mathrm{~m} \times 50 \mathrm{~m}$ butterfly survey plot and they were conducted on the same days as the butterfly surveys. To obtain climatic information we used two National Climate Weather Stations (240775: BigSky, and 486440: Moran $5 \mathrm{WNW}$ ) to represent the GYE study regions. Daily precipitation and temperature data were obtained from these locations and summarized at an annual level. To detect trends in individual species over time, we will analyze the butterfly species for responses in both abundance and distribution across the hydrological gradient. Species with adequate sample sizes will be analyzed individually by meadow type using regressions to test for temporal trends and relationships with annual 
climatic variables (e.g., average daily temperature, and precipitation) as well as host plant cover, and nectar resources.

\section{RESULTS}

Here we provide an archive of some of these long-term data, including maps of the study sites (Figs. 1-2) with UTM locations, area, and

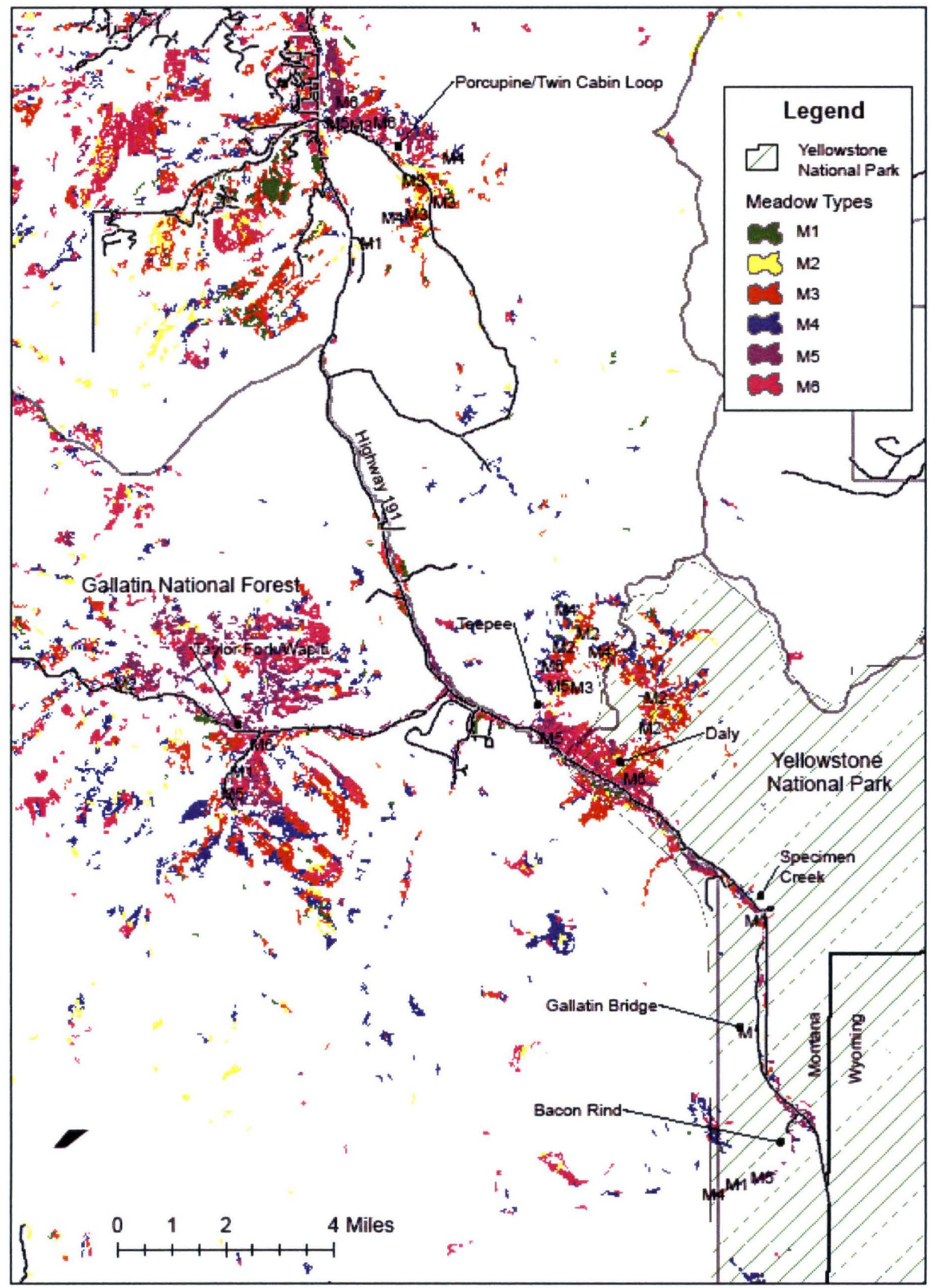

Figure 1. Gallatin Study Region including 30 long-term montane meadow survey sites. M1 meadows are hydric, M3 mesic and M6 meadows are xeric. 


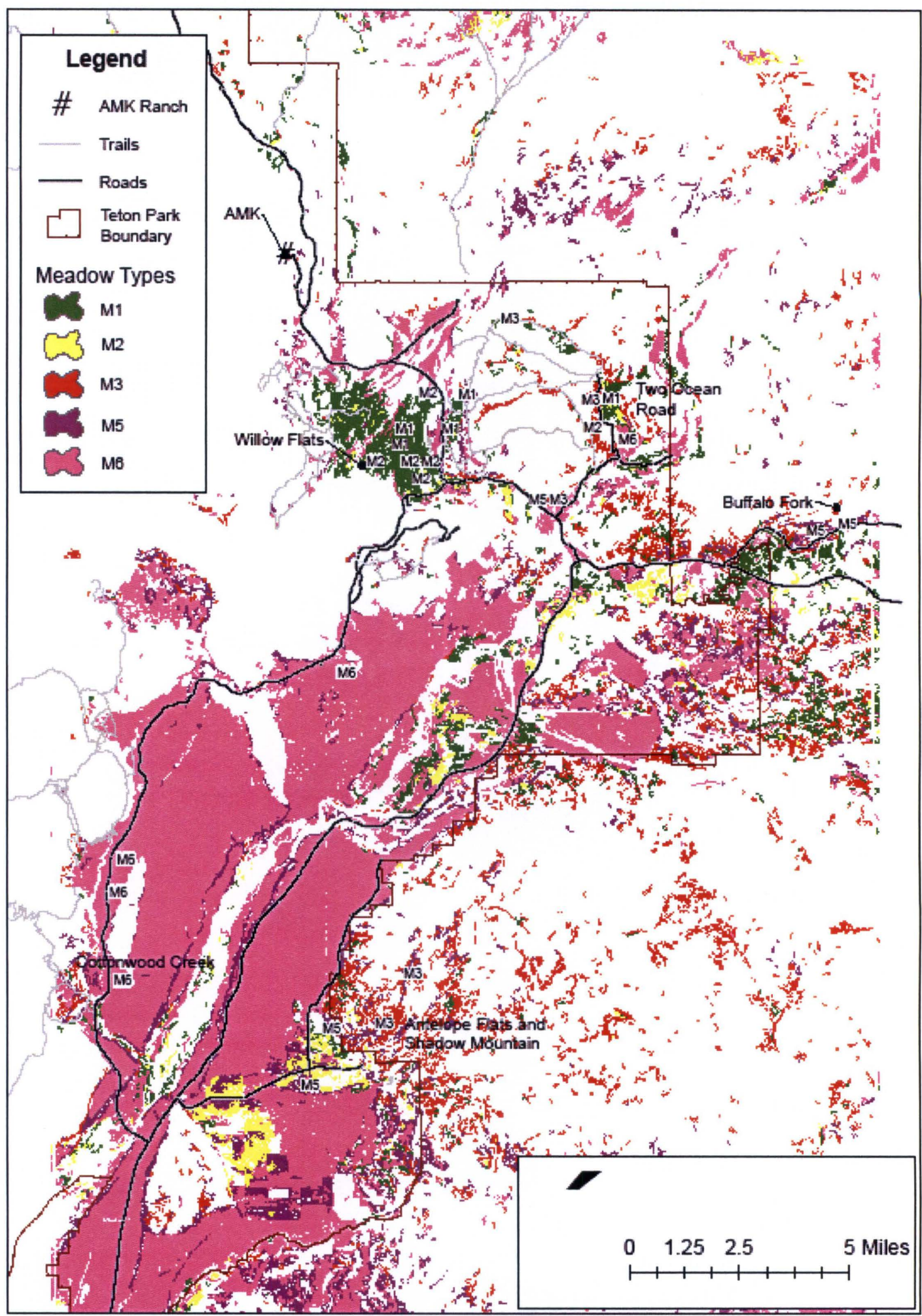

Figure 2. Teton Study Region including 25 long-term montane meadow survey sites. M1 meadows are hydric, M3 mesic and M6 meadows are xeric. M4 meadows are not present in the Teton region. 


\begin{tabular}{|c|c|c|c|c|c|c|}
\hline Region & Site \# & Site Name & $\begin{array}{c}\text { UTM } \\
\text { Northing }\end{array}$ & $\begin{array}{c}\text { UTM } \\
\text { Easting }\end{array}$ & $\begin{array}{c}\text { Elevation } \\
\text { (ft) }\end{array}$ & $\begin{array}{c}\text { Area } \\
\text { (ha) }\end{array}$ \\
\hline \multirow[t]{30}{*}{ Gallatins } & GM1A & Twin Cabin Willows & 5004420 & 482077 & 6424 & 1.63 \\
\hline & GM1B & Bacon Rind & 4975875 & 492784 & 7313 & 1.39 \\
\hline & GM1C & Specimen Creek & 4983802 & 493514 & 6935 & 1.72 \\
\hline & GM1D & Wapiti (Taylor Fork) & 4988439 & 478165 & 7050 & 1.05 \\
\hline & GM1E & Gallatin Bridge & 4979476 & 493792 & 7060 & $\sim 1.00^{* *}$ \\
\hline & GM2A & Taylor Fork & 4991010 & 474842 & 7080 & 4.00 \\
\hline & GM2B & Teepee Wet & 4992350 & 488203 & 7152 & 3.97 \\
\hline & GM2C & Daly South & 4990032 & 490471 & 7047 & 5.73 \\
\hline & GM2D & Figure 8 & 4992079 & 487750 & 7024 & 1.17 \\
\hline & GM2E & Daly North & 4990504 & 490527 & 7109 & 3.02 \\
\hline & GM3A & Porcupine Exclosure & 5007924 & 481627 & 6322 & 2.97 \\
\hline & GM3B & Black Bear Meadow & 5004666 & 483472 & 7001 & 31.88 \\
\hline & GM3C & Porcupine/Twin Meadow & 5005633 & 483988 & 6611 & 19.10 \\
\hline & GM3D & Porcupine Fork & 5006304 & 483518 & $6400^{*}$ & 10.00 \\
\hline & GM3E & V Meadow Teepee Creek & 4990795 & 488003 & 6998 & 15.10 \\
\hline & GM4A & Twin Cabin Pass & 5004902 & 483106 & 6909 & 5.77 \\
\hline & GM4B & Porcupine 1.5 Creek & 5006757 & 484573 & 6680 & 11.60 \\
\hline & GM4C & Teepee & 4992771 & 487906 & 7237 & 11.65 \\
\hline & GM4D & Teepee East Feeder Stream & 4991767 & 488234 & 7231 & 5.54 \\
\hline & GM4E & Bacon Rind & 4975686 & 492264 & 7342 & 2.53 \\
\hline & GM5A & Bacon Rind M5 & 4976074 & 493441 & 7290 & 10.15 \\
\hline & GM5B & Porcupine M5 & 5007844 & 481033 & 6224 & 10.25 \\
\hline & GM5C & Wapiti Cabin & 4987714 & 477806 & 7175 & 10.19 \\
\hline & GM5D & Teepee 191 & 4989358 & 487389 & 6722 & 19.11 \\
\hline & GM5E & Teepee Sage & 4990752 & 487377 & 6883 & 2.48 \\
\hline & GM6A & Porcupine 3rd Creek & 5007623 & 482059 & 6375 & 25.44 \\
\hline & GM6B & Wapiti Pond & 4989242 & 478620 & 6942 & 55.87 \\
\hline & GM6C & Daly & 4988125 & 489568 & $6800^{*}$ & 20.54 \\
\hline & GM6D & Teepee Burn & 4991416 & 487553 & 7047 & 21.09 \\
\hline & GM6E & Gallatin Cabin & 5008449 & 481177 & 6184 & 5.62 \\
\hline
\end{tabular}

* Elevation taken from 7.5 min USGS map

** Estimated area value

Table 1. Study site locations and descriptions for the Gallatin region. UTM (Universal Transverse Mercator) coordinates as well as elevation and area of the meadow each site are listed for each site. Elevation and UTM data are based on readings from GPS (Magellan) during 2006 with accuracy within 20 meters. 


\begin{tabular}{cllcccc}
\hline Region & Site \# & \multicolumn{1}{c}{ Site Name } & $\begin{array}{c}\text { UTM } \\
\text { Northing }\end{array}$ & $\begin{array}{c}\text { UTM } \\
\text { Easting }\end{array}$ & $\begin{array}{c}\text { Elevation } \\
\text { (ft) }\end{array}$ & $\begin{array}{c}\text { Area } \\
\text { (ha) }\end{array}$ \\
\hline Tetons & TM1A & Jackson Lodge Willow North & 4859445 & 533499 & 6863 & $548.08^{*}$ \\
& TM1B & Jackson Lodge Willow South & 4858850 & 533656 & 6830 & $548.08^{*}$ \\
TM1C & Grand View & 4859989 & 534803 & 6883 & 53.65 \\
TM1D & Two Ocean Road & 4859500 & 540316 & 6909 & 77.85 \\
TM1E & Jackson Dam & 4857743 & 532539 & 6811 & $548.08^{*}$ \\
TM2A & Willow Flats North & 4857576 & 533684 & 6801 & 1.75 \\
TM2B & Willow Flats South & 4857071 & 533741 & 6784 & 2.06 \\
TM2C & Two Ocean Road & 4859177 & 539992 & 6988 & 5.82 \\
TM2D & Christian Pond & 4858777 & 534780 & 6853 & 1.64 \\
TM2E & Cygnet Pond & 4860372 & 530345 & 6880 & 1.08 \\
TM3A & Two Ocean Lake & 4862882 & 536736 & 6958 & 7.74 \\
TM3B & Two Ocean Road & 4859928 & 540060 & 6991 & 35.54 \\
TM3C & Lozier Hill & 4856601 & 538763 & 6837 & 12.45 \\
TM3D & Shadow Mountain Hairpin & 4838220 & 532801 & 7851 & 119.62 \\
TM3E & Sound Of Music & 4839404 & 533446 & 8175 & 3.32 \\
TM5A & Lozier Hill & 4856428 & 537905 & 6853 & 13.38 \\
TM5B & Buffalo Fork West & 4855233 & 548289 & 7048 & 54.47 \\
TM5C & Buffalo Fork East & 4855564 & 549366 & 6952 & 6.91 \\
TM5D & Antelope Flats & 4835685 & 528705 & 6745 & 76.03 \\
TM5E & Shadow Mountain Base & 4837479 & 530133 & 6801 & 23.71 \\
TM6A & Two Ocean Road & 4858336 & 540888 & 6886 & 65.78 \\
TM6B & Cow Lake & 4851603 & 532596 & 6926 & 2805.39 \\
TM6C & Timbered Island Northwest & 4838381 & 522773 & 6801 & $4801.85^{* *}$ \\
TM6D & Timbered Island Southwest & 4841752 & 522279 & 6801 & $4801.85^{* *}$ \\
TM6E & Cottonwood Creek & 4838382 & 522770 & 6673 & $4801.85^{* *}$ \\
\hline The & & & & \\
\hline
\end{tabular}

* One M1 meadow

** One M6 meadow

Table 2. Study site locations and descriptions for the Teton Region. UTM (Universal Transverse Mercator) coordinates as well as elevation and area of the meadow each site are listed for each site. Elevation and UTM data are based on readings from GPS (Magellan) during 2006 with accuracy within 20 meters. 
Table 3. Abundance for each butterfly species observed in the Gallatin Region for the years: 1997, 1998, 2000, 2001, 2006, and 2007 totaled over all years, sites and replicates.

\begin{tabular}{|c|c|c|}
\hline Species Latin Names & Species Common Names & $\begin{array}{c}\text { Total } \\
\text { Abundance }\end{array}$ \\
\hline Agriades glandon & Arctic Blue & 39 \\
\hline Anthocharis sara stella & Stella Sara Orangetip & 18 \\
\hline Boloria freija* & Freija Fritillary & 2 \\
\hline Boloria frigga & Frigga Fritillary & 4 \\
\hline Boloria kriemhild & Relict Fritillary & 97 \\
\hline Boloria montinus* & Purplish Fritillary & 1 \\
\hline Boloria selene & Silver-bordered Fritillary & 39 \\
\hline Callophrys sheridanii & Sheridan's Hairstreak & 2 \\
\hline Cercyonis oetus & Small Wood-Nymph & 592 \\
\hline Cercyonis sthenele & Great Basin Wood-Nymph & 1 \\
\hline Chlosyne palla & Northern Checkerspot & 36 \\
\hline Coenonympha haydenii & Hayden's Ringlet & 1579 \\
\hline Coenonympha tullia inornata & Inornate Common Ringlet & 93 \\
\hline Colias alexandra & Queen Alexandra's Sulphur & 2 \\
\hline Colias christina & Christina's Sulphur & 11 \\
\hline Colias eurytheme & Orange Sulphur & 1 \\
\hline Colias gigantea & Giant Sulphur & 17 \\
\hline Colias pelidne & Pelidne Sulphur & 22 \\
\hline Colias philodice & Clouded Sulphur & 61 \\
\hline Danaus plexippus & Monarch & 2 \\
\hline Erebia epipsodea & Common Alpine & 659 \\
\hline Euchloe ausonides & Large Marble & 126 \\
\hline Euphilotes enoptes ancilla & Dotted Blue & 27 \\
\hline Euphydryas chalcedona & Variable Checkerspot & 20 \\
\hline Euphydryas editha & Edith's Checkerspot & 17 \\
\hline Euphydryas gillettii & Gillett's Checkerspot & 18 \\
\hline Glaucopsyche lygdamus & Silvery Blue & 216 \\
\hline Glaucopsyche piasus & Arrowhead Blue & 3 \\
\hline Limenitis weidemeyerii & Weidemeyer's Admiral & 2 \\
\hline Lycaeides idas & Northern Blue & 18 \\
\hline Lycaeides melissa & Melissa Blue & 92 \\
\hline Lycaena cupreus & Lustrous Copper & 1 \\
\hline Lycaena dione* & Gray Copper & 1 \\
\hline Lycaena editha & Edith's Copper & 105 \\
\hline Lycaena helloides & Purplish Copper & 488 \\
\hline Lycaena heteronea & Blue Copper & 160 \\
\hline Lycaena hyllus & Bronze Copper & 2 \\
\hline Lycaena mariposa & Mariposa Copper & 9 \\
\hline Lycaena nivalis & Lilac-bordered Copper & 7 \\
\hline Nymphalis antiopa & Mourning Cloak & 4 \\
\hline Nymphalis californica & California Tortoiseshell & 2 \\
\hline Nymphalis milberti & Milbert's Tortoiseshell & 3 \\
\hline Oeneis chryxus chryxus & Brown Chryxus Arctic & 29 \\
\hline Oeneis uhleri & Uhler's Arctic & 1 \\
\hline
\end{tabular}


Table 3. (continued)

\begin{tabular}{|c|c|c|}
\hline Species Latin Names & Species Common Names & $\begin{array}{c}\text { Total } \\
\text { Abundance }\end{array}$ \\
\hline Papilio eurymedon & Pale Swallowtail & 1 \\
\hline Papilio machaon * & Old World Swallowtail & 5 \\
\hline Papilio rutulus & Western Tiger Swallowtail & 2 \\
\hline Papilio zelicaon & Anise Swallowtail & 21 \\
\hline Parnassius clodius & Clodius Parnassian & 53 \\
\hline Parnassius phoebus smintheus & Rocky Mountan Phoebus Parnassian & 121 \\
\hline Phyciodes campestris & Field Crescent & 504 \\
\hline Phyciodes mylitta* & Mylitta Crescent & 107 \\
\hline Phyciodes selenis & Northern Crescent & 27 \\
\hline Phyciodes tharos & Pearl Crescent & 13 \\
\hline Pieris napi marginalis & Margined Mustard White & 83 \\
\hline Pieris napi oleracea & Mustard White & 27 \\
\hline Pieris rapae & Cabbage White & 3 \\
\hline Plebejus icarioides & Boisduval's Blue & 726 \\
\hline Plebejus lupini & Lupine Blue & 40 \\
\hline Plebejus saepiolus & Greenish Blue & 366 \\
\hline Plebejus shasta & Shasta Blue & 13 \\
\hline Polygonia faunus* & Green Comma & 4 \\
\hline Polygonia gracilis* & Hoary Comma & 2 \\
\hline Polygonia satyrus* & Satyr Comma & 1 \\
\hline Pontia beckerii & Becker's White & 9 \\
\hline Pontia occidentalis & Western White & 7 \\
\hline Pontia protodice & Checkered White & 19 \\
\hline Satyrium titus* & Coral Hairstreak & 1 \\
\hline Speyeria atlantis hesperis & Hesperis Atlantis Fritillary & 95 \\
\hline Speyeria callippe & Callippe Fritillary & 37 \\
\hline Speyeria cybele & Great Spangled Fritillary & 1 \\
\hline Speyeria egleis & Great Basin Fritillary & 15 \\
\hline Speyeria hydaspe & Hydaspe Fritillary & 8 \\
\hline Speyeria mormonia & Mormon Fritillary & 879 \\
\hline Speyeria zerene & Zerene Fritillary & 21 \\
\hline Vanessa cardui & Painted Lady & 98 \\
\hline
\end{tabular}

* Butterfly Species only found only in Gallatin Region butterfly surveys. 
Table 4. Abundance for each butterfly species observed in the Teton Region for the years: 1997, 1998, 2000, 2001, 2003-2007 totaled over all years, sites and two replicates.

\begin{tabular}{|c|c|c|}
\hline Species Latin Names & Species Common Names & $\begin{array}{c}\text { Total } \\
\text { Abundance }\end{array}$ \\
\hline Agriades glandon & Arctic Blue & 45 \\
\hline Anthocharis sara stella & Stella Sara Orangetip & 10 \\
\hline Boloria frigga & Frigga Fritillary & 67 \\
\hline Boloria kriemhild & Relict Fritillary & 74 \\
\hline Boloria selene & Silver-bordered Fritillary & 167 \\
\hline Callophrys dumetorum ** & Bramble Hairstreak & 18 \\
\hline Callophrys sheridanii & Sheridan's Hairstreak & 13 \\
\hline Cercyonis oetus & Small Wood-Nymph & 492 \\
\hline Cercyonis pegala** & Common Wood-Nymph & 2 \\
\hline Chlosyne palla & Northern Checkerspot & 55 \\
\hline Coenonympha haydenii & Hayden's Ringlet & 692 \\
\hline Coenonympha tullia inornata & Inornate Common Ringlet & 593 \\
\hline Colias alexandra & Queen Alexandra's Sulphur & 6 \\
\hline Colias christina & Christina's Sulphur & 4 \\
\hline Colias eurytheme & Orange Sulphur & 2 \\
\hline Colias gigantea & Giant Sulphur & 69 \\
\hline Colias interior ${ }^{* *}$ & Pink-Edged Sulphur & 17 \\
\hline Colias pelidne & Pelidne Sulphur & 26 \\
\hline Colias philodice & Clouded Sulphur & 42 \\
\hline Erebia epipsodea & Common Alpine & 605 \\
\hline Euchloe ausonides & Large Marble & 80 \\
\hline Euphilotes enoptes ancilla & Dotted Blue & 73 \\
\hline Euphydryas chalcedona & Variable Checkerspot & 45 \\
\hline Euphydryas editha & Edith's Checkerspot & 24 \\
\hline Euphydryas gillettii & Gillett's Checkerspot & 6 \\
\hline Euptoieta claudia* & Variegated Fritillary & 1 \\
\hline Glaucopsyche lygdamus & Silvery Blue & 101 \\
\hline Glaucopsyche piasus & Arrowhead Blue & 29 \\
\hline Limenitis weidemeyerii & Weidemeyer's Admiral & 12 \\
\hline Lycaeides idas & Northern Blue & 56 \\
\hline Lycaeides melissa & Melissa Blue & 62 \\
\hline Lycaena cupreus & Lustrous Copper & 13 \\
\hline Lycaena editha & Edith's Copper & 81 \\
\hline Lycaena helloides & Purplish Copper & 239 \\
\hline Lycaena heteronea & Blue Copper & 475 \\
\hline Lycaena hyllus & Bronze Copper & 37 \\
\hline Lycaena nivalis & Lilac-bordered Copper & 68 \\
\hline Lycaena phlaeas ${ }^{\star \star}$ & American Copper & 1 \\
\hline Lycaena rubidus ${ }^{\star *}$ & Ruddy Copper & 1 \\
\hline Nymphalis antiopa & Mourning Cloak & 7 \\
\hline Nymphalis californica & California Tortoiseshell & 1 \\
\hline Nymphalis milberti & Milbert's Tortoiseshell & 7 \\
\hline Oeneis chryxus chryxus & Brown Chryxus Arctic & 12 \\
\hline Oeneis jutta** & Jutta Arctic & 3 \\
\hline
\end{tabular}


Table 4. (continued)

\begin{tabular}{|c|c|c|}
\hline Species Latin Names & Species Common Names & $\begin{array}{c}\text { Total } \\
\text { Abundance }\end{array}$ \\
\hline Oeneis uhleri & Uhler's Arctic & 1 \\
\hline Papilio canadensis ** & Canadian Tiger Swallowtail & 7 \\
\hline Papilio eurymedon & Pale Swallowtail & 3 \\
\hline Papilio rutulus & Western Tiger Swallowtail & 21 \\
\hline Papilio zelicaon & Anise Swallowtail & 27 \\
\hline Parnassius clodius & Clodius Parnassian & 38 \\
\hline Parnassius phoebus & Rocky Mountan Phoebus & \\
\hline smintheus & Parnassian & 9 \\
\hline Phyciodes campestris & Field Crescent & 79 \\
\hline Phyciodes selenis & Northern Crescent & 266 \\
\hline Phyciodes tharos & Pearl Crescent & 5 \\
\hline Pieris napi marginalis & Margined Mustard White & 42 \\
\hline Pieris napi oleracea & Mustard White & 7 \\
\hline Pieris rapae & Cabbage White & 5 \\
\hline Plebejus icarioides & Boisduval's Blue & 1305 \\
\hline Plebejus Iupini & Lupine Blue & 202 \\
\hline Plebejus saepiolus & Greenish Blue & 827 \\
\hline Plebejus shasta & Shasta Blue & 21 \\
\hline Pontia beckerii & Becker's White & 3 \\
\hline Pontia occidentalis & Western White & 5 \\
\hline Pontia protodice & Checkered White & 17 \\
\hline Satyrium sylvinus & Coral Hairstreak & 1 \\
\hline Speyeria atlantis hesperis & Hesperis Atlantis Fritillary & 6 \\
\hline Speyeria callippe & Callippe Fritillary & 126 \\
\hline Speyeria cybele & Great Spangled Fritillary & 41 \\
\hline Speyeria edwardsii & Edwards's Fritillary & 3 \\
\hline Speyeria egleis & Great Basin Fritillary & 10 \\
\hline Speyeria hydaspe & Hydaspe Fritillary & 8 \\
\hline Speyeria mormonia & Mormon Fritillary & 596 \\
\hline Speyeria zerene & Zerene Fritillary & 28 \\
\hline Vanessa annabella** & West Coast Lady & 1 \\
\hline Vanessa atalanta** & Red Admiral & 2 \\
\hline Vanessa cardui & Painted Lady & 74 \\
\hline
\end{tabular}

** Butterfly Species only found only in Teton Region butterfly surveys.

\section{CONCLUSIONS}

The benefits of a long-term data set increase our probability of detecting climate driven population fluctuations. Many studies on butterfly populations only observe the communities for two to three years and extrapolate trends with little assurance that their observed patterns identify community patterns (Hill et al., 1995; Spitzer et al., 1997; Gutierrez and Menendez, 1998). Previous work in the GYE system has shown that the different meadow types have distinct plant communities (Jakubauskas et al., 2001; Kindscher et al., 1998) as well as predictable butterfly communities that associate with each of these meadows established along a hydrologic gradient (Debinski et al., 2002). Recent analysis of the plant community in our study sites shows that the forb cover in many of the meadow types has decreased from 1997 to 2007 (Debinski, unpublished data), particularly in the mesic to xeric meadow types. Butterfly communities may also be showing shifts (Debinski, unpublished data). Our next steps 
will be to analyze butterfly trends with respect to changes in both the plant cover and abiotic data such as temperature and precipitation. Understanding climatic influences on butterfly communities will provide a window into understanding larger ecosystem responses to long-term drought in the GYE

\section{$\downarrow$ Literature Cited}

Binzenhofer, B., B. Schroder, B. Strauss, R. Biedermann and J. Settele. 2005. Habitat models and habitat connectivity analysis for butterflies and burnet moths - the example of Zygaena carniolica and Coenonympha arcania. Biological Conservation 126: 247 259.

Cross-Smith, M. and J. Hart. 2007. Compensatory responses to loss of warming-sensitive plant species. Ecology 88:740-748.

Debinksi, D. M., C. Ray and E.H. Saveraid. 2001. Species diversity and the scale of the landscape mosaic:do scales of movement and patch size affect diversity? Biological Conservation 98:179-190.

Debinski, D. M. and Brussard, P.F. 1994. Using biodiversity data to asses species-habitat relationships in Glacier National Park, Montana. Ecological Applications 4: $833-$ 843.

Debinski, D.M., M.E. Jakubauskas and K. Kindscher. 2000. Montane meadows as indicators of environmental change. Environmental Monitoring and Assessment 64: 213-225.

Debinski, D.M., R.E. VanNimwegen and M.E. Jakubauskas. 2006. Quantifying relationships between bird and butterfly community shifts and environmental change. Ecological Applications 16 (1): 380-393.

Debinski, D.M., M.E. Jakubauskas, K. Kindscher, E.H. Saveraid and M. Borgognone. 2002. Predicting meadow communities and species occurrences in the Greater Yellowstone Ecosystem. P. 499-506. In Predicting Species Occurrences: Issues of Scale and
Accuracy (Scott, J.M., P.J. Heglund, M. Morrison, M. Raphael, J. Haufler, B. Wall, editors). Island Press. Covello, CA.

Dennis, R.L.H., T.G. Shreeve, N.J.B. Isaac, P.B. Hard, R. Fox and J. Asher. 2006. The effects of visual apparency on bias in butterfly recording and monitoring. Biological Conservation 128: 486-492.

DeValpine, P. and J. Harte. 2001. Plant response to experimental warming in a mountain meadow. Ecology 82: 637-648.

Field, R.G., T. Gardiner, C.F. Mason, and J. Hill. 2006. Countryside stewardship scheme and butterflies: a study of plant and butterfly species richness. Biodiversity and Conservation 15:443-452.

Grill, A. and D.F.R. Cleary. 2003. Diversity patterns in butterfly communities of the Greek native reserve Dadia. Biological Conservation 114: 427-436.

Gutierrez, D. and R. Menendez. 1998. Stability of butterfly assemblages in relation to the level of numerical resolution and altitude. Biodiversity and Conservation 7: 967-979.

Hill, J. K., C. Hamer, L.A. Lace and W.M.T. Bunham. 1995. Effects of selective logging on tropical forest butterflies on Buru Indoneisa. Journal of Applied Ecology 32: 754-760.

Kindscher K., A. Fraser, M.E Jakubauskas, and D.M. Debinski. 1998. Identifying wetland meadows in Grand Teton National Park using remote sensing and average wetland values. Wetlands Ecology and Management 5:265-273.

Jakubauskas, M.E., D.M. Debinski and K. Kindscher. 1996. Integration of multispectral and ecological data for characterizing montane meadow communities in the Greater Yellowstone Ecosystem. IEEE 2300-2302.

Jakubauskas, P., K. Kindscher, and D.M. Debinski. 2001 Hyperspectral remote sensing of nonforested montane vegetation communities. Third International 
Conference on Geospatial Information in Agriculture and Forestry. Denver, Co. Nov 5-7.

Lawton, J.H. and K.J. Gaston 1989. Temporal patterns to the herbivorous insects of bracken: a test of community predictability. Journal of Animal Ecology 58: 1021-1034.

Nally, R.M., E. Fleishman, J.P. Fay and D.D. Murphy. 2003. Modeling butterfly species richness using mesoscale environmental variables: model construction and validation for mountain ranges in the Great Basin in Western North America. Biological Conservation 110: 21-31.

Ockinger, E., 0. Hammerstedt, S.G. Nilsson and H.G. Smith. 2006. The relationship between local extinctions of grassland butterflies and increased soil nitrogen levels. Biological Conservation 128: 564-573.

Parmesan, C. 2006. Ecological and evolutionary responses to recent climate change. Annual Review of Ecological. Systematics. 37: 637669 .

Pimm, S.L. 1984. Complexity and stability of ecosystems. Nature 307: 321-326.

Rahel, F.J. 1990. The hierarchical nature of community persistence: a problem of scale. American Naturalist 136: 328-344.
Saavedra, F., D.W. Inouye., M.V. Price and J. Harte. 2003 Changes in flowering and abundance of Delphinium nuttallianum (Ranunculaceae) in response to a subalpine climate warming experiment. Global Change Biology 9: 885-894.

Scalercio, S.R. Pizzollotto and P. Brandmayr. 2007. Multi-scale analysis of butterfly diversity in a Mediterranean mountain landscape: mapping and evaluation of community vulnerability. Biodiversity and Conservation 16(12): 3463-3479.

Simonson, S.E., P.A. Opler, T.J. Stohlgren and G.W. Chong. 2001. Rapid assessment of butterfly diversity in a montane landscape. Biodiversity and Conservation 10: 13691386.

Spitzer, K.,J. Jaros, J. Havelka, and J. Leps. 1997. Effects of small-scale disturbance on butterfly communities of an Indochinese montane rainforest. Biological Conservation 80: 9-15.

Su, J.C., D.M. Debinski and K. Kindscher. 2004. Beyond species richness: Community similarity as a measure of cross taxon congruence for Coarse-Filter Conservation. Conservation Biology 18: 167-173.

Thomas, C.D. and I.S.G. Abery. 1995. Estimating rates of butterfly decline from distribution maps: the effects of scale. Biological Conservation 73: 57-65.

Weaver, J.E. 1958. Classification of root systems of forbs of grassland and a consideration of their significance. Ecology 39 (3): 393-401. 